\title{
A Modified Genetic based Neural Network Model for Online Character Recognition
}

\author{
J. O. Adigun \\ Department of \\ Computer Technology, \\ Yaba College of \\ Technology, \\ Lagos, Nigeria
}

\author{
E. O. Omidiora \\ Department of \\ Computer Science and \\ Engineering \\ Ladoke Akintola \\ University of \\ Technology, \\ Ogbomoso, Nigeria
}

\author{
S. O. Olabiyisi \\ Department of \\ Computer Science and \\ Engineering \\ Ladoke Akintola \\ University of \\ Technology \\ Ogbomoso, Nigeria
}

\author{
O. D. Fenwa \\ Department of \\ Computer Science and \\ Engineering \\ Ladoke Akintola \\ University of \\ Technology \\ Ogbomoso Nigeria
}

\begin{abstract}
Character Recognition has become an intensive research areas during the last few decades because of its potential applications. However, most existing classifiers used in recognizing handwritten online characters suffer from poor feature selection and slow convergence which affect training time and recognition accuracy. This paper proposed a methodology that is based on extraction of structural features (invariant moment, stroke number and projection) and a statistical feature (zoning) from the characters. A genetic algorithm was modified through its fitness function and genetic operators to minimize the character recognition errors. The Modified Genetic Algorithm (MGA) was used to select optimized feature subset of the character to reduce the number of insignificant and redundant features. A genetic based neural network model was developed by integrating the MGA into an existing Modified Optical Backpropagation (MOBP) learning algorithm to train the network. Three classifiers $(\mathrm{C} 1$, $\mathrm{C} 2$ and $\mathrm{C} 3$ ) were then formulated from MGA-MOBP such that $\mathrm{C} 1$ classified without using MGA at classification level, C2 classified using MGA at classification level while C3 employed MGA at feature selection level and classified at classification level The developed C3 achieves a better performance of recognition accuracy and recognition time.
\end{abstract}

\section{General Terms}

Pattern Recognition

\section{Keywords}

Artificial Neural Network, optical backpropagation, genetic algorithm, character recognition, feature extraction, feature selection, genetic operators.

\section{INTRODUCTION}

Tremendous research efforts have been devoted to online character recognition that is used to translate human readable characters to machine-readable codes.

Development in Information Technology has produced varieties of electronics devices such as Personal Digital Assistants (PDAs), handheld computers where non-keyboard based method of data entry are receiving more attention in the research of pattern recognition. The increase in usage of handheld devices has created a growing demand for algorithm that would reduce the processing time and increase recognition accuracy [8][10][[12]. Character recognition can be divided into two categories namely: Optical Character
Recognition (OCR) and Intelligent Character Recognition (ICR). OCR (offline)recognition for which recognition takes place on a static image captured once the writing process is over [1][4][9] whereas in ICR (online), handwriting data are captured during writing, the two dimensional coordinates of successive points are represented as function of time and information on the ordering of strokes are also available [16]. However, optimization techniques can be integrated into classification techniques in character recognition systems to further improve the overall performance of the recognition system. The backpropagation neural network is a popular algorithm for training a multilayer network, despite this, it has a lot of limitations: slow convergence and easily be trapped in local minimal. Hence, this paper integrates the modified genetic algorithm and modified optical backpropagation to achieve a better performance of training time and recognition accuracy. Three classifiers C1 (MGA and MOBP), C2 (MGABased MOBP) and C3 (MGA and MGABased MOBP) were formulated from MGA-MOBP to check the performance of the system. Section two describes related work, while section three contains the materials and methods and draw conclusion in section four.

\section{RELATED WORK}

Online character recognition has been one of the most challenging research areas in the field of pattern recognition in the recent years[1]. The selection of appropriate feature extraction is undoubtedly the single most important factor in achieving high recognition performance [11][7]. [8] proposed a method based on the extraction of different spatial and temporal features from strokes of the character and recognition was done by using genetic algorithm as a tool to find an optimal subset of the stroke features. Recognition rate of $83.1 \%$ was achieved. [14] developed English character recognition system using the hybrid of standard backpropagation and genetic algorithm for recognition of uppercase alphabets. Recognition performance was $91.1 \%$. [9] designed a novel and robust hybrid recognition system for Odia handwritten character, based on the algorithm of feed forward BPNN combined with Genetic Algorithm (GA) to perform the optimum feature extraction and recognition. [4][5] a hybrid feature extraction technique was developed using geometrical and statistical features. However, a multiple classification character recognition scheme was employed using modified counter propagation network (MCPN) and modified optical backpropagation (MOBP) neural network. 
[17] presented a backpropagation network algorithm combined with genetic algorithm to achieve both accuracy and training swiftness for recognizing alphabets. Recently, researchers have invested in improving backpropagation(BP) algorithm in many ways such as adjustment of learning rate [3], and error adjustment [4][5], However, these works were yet to deliver the desired results. Hence, the aim of this paper is to integrate optimization algorithm (genetic algorithm) into modified backpropagation neural network to improve the performance of system in terms training time and recognition accuracy.

Table 2.1 Comparison Analysis of Existing Techniques

\begin{tabular}{|c|c|c|c|c|}
\hline S/N & Author(s) & Strategy & Limitations & $\begin{array}{c}\text { Performance } \\
\%\end{array}$ \\
\hline 1 & $\begin{array}{c}\text { Abed et al } \\
(2010)\end{array}$ & $\begin{array}{c}\text { Genetic } \\
\text { Algorithm }\end{array}$ & $\begin{array}{c}\text { Slow } \\
\text { training time }\end{array}$ & 95 \\
\hline 2 & $\begin{array}{c}\text { Ranpreet } \text { et al } \\
(2011)\end{array}$ & $\begin{array}{c}\text { Standard } \\
\text { GA, BP } \\
\text { (English } \\
\text { character) }\end{array}$ & $\begin{array}{c}\text { Scaling, } \\
\text { local minima } \\
\text { problems. }\end{array}$ & 91 \\
\hline 3 & $\begin{array}{c}\text { Yeremia } \text { et al } \\
(2012)\end{array}$ & $\begin{array}{c}\text { Standard } \\
\text { BPN, GA }\end{array}$ & $\begin{array}{c}\text { Not } \\
\text { Reported }\end{array}$ & 90.77 \\
\hline 4 & $\begin{array}{c}\text { Padhi } \text { et al } \\
(2012)\end{array}$ & $\begin{array}{c}\text { SBP, } \\
\text { SGA }\end{array}$ & $\begin{array}{c}\text { Local } \\
\text { minima and } \\
\text { loss of } \\
\text { solution }\end{array}$ & 94 \\
\hline 5 & $\begin{array}{c}\text { Fenwa } \text { et al } \\
(2012)\end{array}$ & $\begin{array}{c}\text { MCPN, } \\
\text { MOBP }\end{array}$ & $\begin{array}{c}\text { Long } \\
\text { training time }\end{array}$ & 99 \\
\hline
\end{tabular}

\section{MATERIAL AND METHODS}

Figure 1 shows the block diagram of the character recognition system. The feature selection was used to reduce the number of features by elimination of insignificant and redundant features which improves the classification accuracy. In this paper, a MGA and MOBP model was developed for better online character recognition. To substantiate the effectiveness of the developed algorithm, three types of classifiers, C1, C2 and C3 were formulated. Table 2.1 represents performance evaluation of the existing techniques with related works in literature. It can be deduced from the table that techniques used by Fenwa et al (2012)is better than other techniques. This shows that the performance of Counterpropagation and Optical backpropagation neural network was better than that of Standard backpropagation (Figure 2). The proposed technique will use modified genetic algorithm to optimize the existing modified optical backpropagation nueral network as a better approach to improve the performance of character recognition system in terms of recognition accuracy and recognition time.



\subsection{Feature Extraction}

In this research work, the process of features extraction were employed to determine the most optimal set of features for character recognition system. The features used were based on stroke information, invariant moments, projection and zoning of the handwritten character to create a global feature vector. Most of the techniques used only one property feature. This research combines structural and statistical features to complement each other, handle style variations and distinguish one input pattern from another pattern to achieve better performance. Hence, a hybrid was developed to highlight different character properties that effectively identity a character as shown in Figure 3.

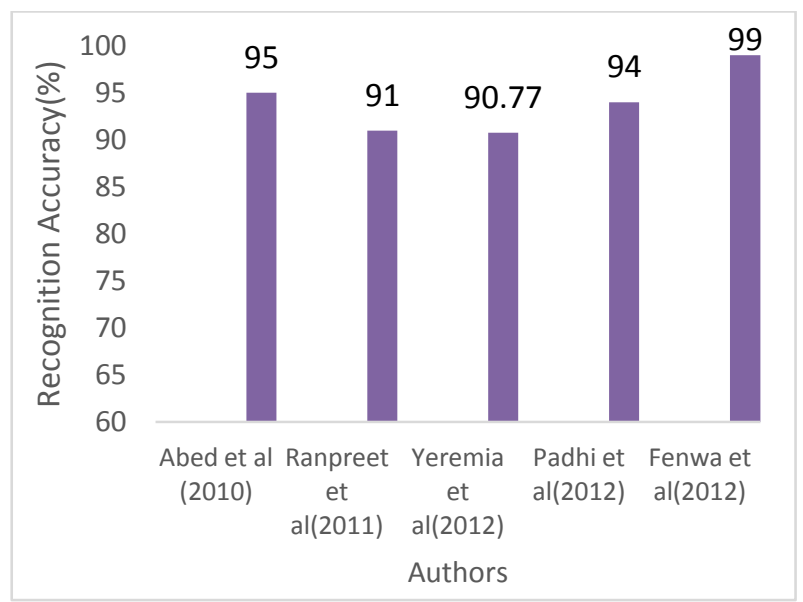

Figure 2 Comparison Analysis of Existing Techniques of Table 2.1

\section{Developed Hybrid (Struct-Statistical) Feature Extraction Algorithm}

The following stages were taken in the development:

Step 1: Stroke information of the image characters was obtained this include: pressure used, number of strokes and projection count of the character.

Step 2: Apply invariant moments to determine position, size and orientation of the character was applied 
Step 3: Run Hybrid Zoning Algorithm on the character.

Step 4: Apply feature selection to reduce number of features by elimination of insignificant features.

Step 5: The outputs of the extracted and selected features of the characters was fed into the digitization stage in order to convert all the extracted features into digital forms.

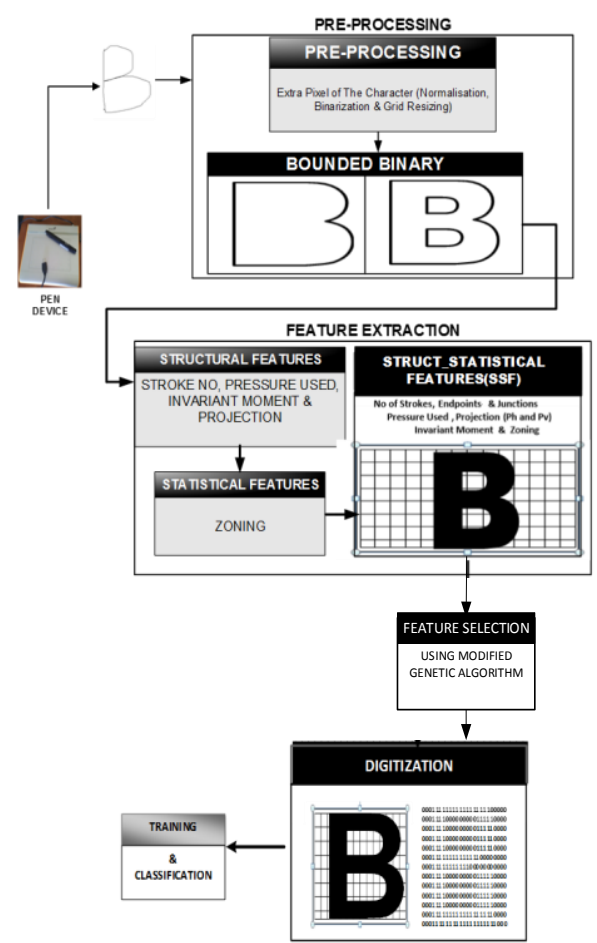

Figure 3: Developed Feature Extraction Model

\section{Hybrid Zoning Algorithm:}

In this paper a hybrid of modified ICZ and modified ZCZ proposed by Fenwa et al,2012 was used:

Input: Preprocessed character image

Output: Features for Classification and Recognition

Begin

Step 1: Divide the input image into 25 equal zones

Step 2: Compute the input image centroid

Step 3: Compute the distance between the image centroid to each pixel present in the zone

Step 4: Repeat step 3 for the entire pixel present in the zone

Step 5: Compute average distance between these points

Step 6: Compute the zone centroid

Step 7: Compute the distance between the zone centroid to each pixel present in the zone.

Step 8: Repeat step 7 for the entire pixel present in the zone

Step 9: Compute average distance between these points

Step 10: Repeat the steps 3-9 sequentially for the entire zones

Step11:Finally, $2 * n(50)$ such features are obtained for classification and recognition.

\section{Modified Genetic Algorithm (MGA)}

The training of Neural network by backpropagation is time consuming and slow convergence due to local minimal. The loss of potential solution by standard genetic algorithm (SGA) is a common drawback. To overcome these problems, it is therefore necessary to modify standard genetic algorithm to speed up the convergence and reduce computational time for enhancing the training capacity of the network. To improve the SGA optimization speed, two unique approaches were modified.

(a) The main idea of Genetic Algorithm is to mimic the processes in nature, even though selection and crossover operators effectively work, there is a possibility that SGA may lose some potentially useful information. That is why mutation is needed to prevent falling of all solutions into local optimum of character recognition. The genetic algorithm mutation operator was processed first, followed by selection and crossover. This led to a new modification denoted as MSC(Mutation, Selection Crossover). After mutation, the fitness value for offspring are calculated and best fittest individuals are selected to replace the parent and reproduction of new chromosome.

(b) Modification of Fitness function. To determine the optimum sub solution, it should be noted that fitness function was modified returned as (equation 3.1) is the number of selected zero or approximately zero features from the algorithm and multiplied with the percentage of feature used. The selection of parent was based on best fittest (i.e. smaller fitness values) because the optimization purpose was to reduce the percentage of recognition error while reducing the number of features used[11] and the fitness function was modified as:

Fitness $=($ no of selected zero features $) * \%$ FeatureUsed $) \quad 3.1$

where $\mathrm{H}=$ Hprojection, $\mathrm{DH}=$ Downsample_Height

DW $=$ Downsample_Width

$$
\text { FeatureUsed }=\frac{\mathrm{H}}{\mathrm{DH} * \mathrm{DW}} * 100
$$

The MGA is terminated when some criteria are satisfied, e.g. when a particular point in the search space is encountered.

\subsection{Feature Selection (FS)}

The aim is to reduce the number of features by elimination of insignificant and redundant features which improve the classification accuracy. This reduces the computational burden imposed by using many features and improve the training time and testing efficiency. In the research, modified genetic algorithm is proposed for feature selection. MGA initially starts with a number of solutions known as population. These solutions are represented using a string coding of fixed length. After evaluating each chromosome using a fitness function and assigning a fitness value, three different operators such as mutation, selection and crossover are used to update the population. A repetition of these three operators is known as a generation. The new chromosome will then replace the chromosome with the lowest survival rate. This process will be iterated until the desired error rate is achieved. The features with the bit value " 1 " are selected and the features with the bit value of " 0 ", the corresponding one are rejected

End. 


\section{Feature Selection (Using Modified Genetic Algorithm)}

1) Start

2) Get character features set

3) Initialize parameters (set gen $<=$ number of generation ; set $\mathrm{n}<=$ population size, mutation prob)

4) Generate randomly distributed chromosomes to form initial population

5) Encode features by a chromosome \{using bit strings encoding

6) Gencount $<=0$

7) Rank chromosomes based on its uniqueness \{i.e. first chromosome occurrence $=1$, subsequence occurrence $=$ $0\}$

8) Arrange chromosomes based on their fitness value\{ Accept features with bit value $=1$ and reject features with bit value $=0\}$

9) Mutate selected chromosomes based on mutation probability

10) Select chromosome with best fitness value

11) Perform crossover on parent chromosomes to form new offspring and replace the weak chromosomes with the new offspring

12) gencount $<=$ gencount +1 and repeat until total number of generation

13) if gencount $=$ gen then goto 14 else 7

14) Output chromosome with the highest fitness value(feature selected)

\subsection{Development of Hybrid MGA and MOBP Model}

In this paper, hybrid of modified Genetic Algorithm and modified optical backpropagation neural network was developed as shown in Figure 4. The online character written is first captured using digitizer to get new image for processing. Then the characters are resized for training the neural network which has undergone various processes like converting the grayscale, binarizing, finding edge for feature extraction etc. During the preprocessing and feature extraction, the individual strokes are resampled to make sample data equidistant (standard size). This helps to reduce the variations, gap and blurred in the characters and to avoid anomalous cases. Features are extracted from user's input pattern. The initial inputs of the characters provided are trained with different parameters and different sets of characters.

The characters are classified using three classifiers: $\mathrm{C} 1, \mathrm{C} 2$, and C3. During training, the weights of the network are iteratively adjusted to minimize the error function. The set of outputs obtained are fed into the genetic algorithm to select the best fittest and best solution. The outputs of the genetic algorithm are sent to the neural networks as input. The genetic based neural network model was developed by integrating the MGA into existing MOBP was trained to recognize different types of handwritings. This is achieved by using highly efficient supervised learning algorithm. The output obtained from the trained are stored as files. Match the introduced character with the one in the database template and classify the given character or pattern image. The genetic algorithm is used for optimal feature selection. The extracted features are classified according to identify pattern to which it has been trained.

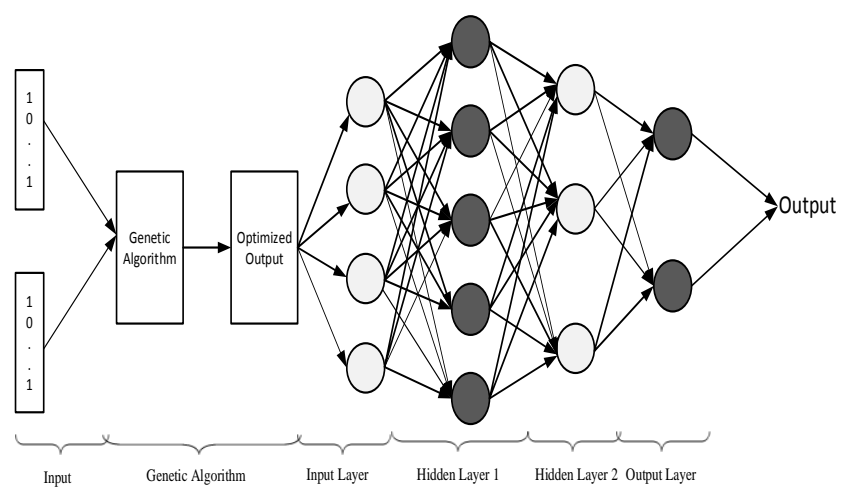

Figure 4: The Hybrid of GA and MOBP Model Used

\subsubsection{The Genetic and Neural Network Algorithm}

This research work employed hybrid of modified Genetic Algorithm and Modified Optical Backpropagation neural networks for the training and classification of the input pattern. The training algorithm involves the following two stages:

Stage A: Performs the training of the character sample generated using Genetic Algorithm.

Step 1: Select character samples collected from various people (Generate random population of $\mathrm{n}$ chromosomes this corresponds to initial population for the genetic Algorithm).

Step 2: Evaluate the fitness function of the population equation

Step 3: Mutate new offspring at each locus based on mutation probability.

Step 4: Select parent chromosomes from the population according to their fitness function (better fitness, bigger chance to be selected).

Step 5: Perform crossover on two strongest parents to create a new offspring and replace the weakest parent with the new child created. Insert the new offspring in the new population and use new generated population for a further run of the algorithm.

STAGE B: Performs the training of the input from the genetic algorithm to the output layer. According to Sutojo et al., (2011). Assume there are $m$ input units, $n$ hidden units, and $p$ output units. $\mathrm{W}_{\mathrm{ji}}^{\mathrm{h}}$ weight on the connection from the $i$ th input unit to $j$ th hidden unit.

1. Input vector, $\mathrm{X}_{\mathrm{p}}=\mathrm{X}_{\mathrm{p} 1}+\mathrm{X}_{\mathrm{p} 2}+\mathrm{X}_{\mathrm{p} 3} \ldots+\mathrm{X}_{\mathrm{pm}}$ to the input units. Each value in input layer receive $X_{p i}$ and forward to values in hidden layer.

2. Calculate the net-input values to the hidden layer units.

3. Calculate the outputs from the hidden layers.

4. Calculate the net-input values to the output layer units.

5. Calculate the outputs from the output units.

6. Calculate the error term for output unit 
i. $\quad$ Modified $\delta^{\mathrm{o}}{ }_{\mathrm{pk}}=3\left(\left(1+\mathrm{e}^{\mathrm{t}}\right)^{2} \cdot \mathrm{f}^{\mathrm{o}^{\prime}}{ }_{k}\left(\right.\right.$ net $\left.\left.^{\mathrm{o}}{ }_{\mathrm{pk}}\right)\right)$

$$
\text { If }\left(Y_{p k}-O_{p k}\right) 2>=0
$$

ii. $\quad$ Modified $\delta^{\mathrm{o}}{ }_{\mathrm{pk}}=-3\left(\left(1+\mathrm{e}^{\mathrm{t}}\right)^{2} \cdot \mathrm{f}^{\mathrm{o}}{ }_{k}\left(\right.\right.$ net $\left.\left.^{\mathrm{o}}{ }_{\mathrm{pk}}\right)\right)$

$$
\operatorname{If}\left(Y_{p k}-O_{p k}\right) 2<=0
$$

iii. Using cubic error adjustment where

$Y_{p k}=$ Desired output

$O_{p k}=$ Network output

$\mathrm{T}=\left(Y_{p k}-O_{p k}\right) 2$

7. Calculate the error term for the hidden units, through applying Modified $\delta_{\mathrm{pk}}^{\mathrm{o}} \quad$ also Modified $\delta^{\mathrm{o}}{ }_{\mathrm{pj}}=\mathrm{f}^{\mathrm{h}^{\prime}}{ }_{j}\left(\right.$ net $\left.^{\mathrm{h}}{ }_{\mathrm{pj}}\right) \cdot\left(\sum_{k=1}^{M}\right.$ Modified $\left.\delta^{\mathrm{o}}{ }_{\mathrm{pk}} \cdot \mathrm{W}^{\mathrm{o}}{ }_{\mathrm{kj}}\right) 3.3$

$\delta^{\mathrm{o}}{ }_{\mathrm{pk}}$ is signal error term for the $k$ th output unit, $\delta^{\mathrm{h}}{ }_{\mathrm{pj}}$ is signal error term for the $j$ th output unit.

8. Update weights on the output layer with learning rate $\eta$

$$
\mathrm{W}^{\mathrm{o}}{ }_{\mathrm{kj}}(t+1)=\mathrm{W}^{\mathrm{o}}{ }_{\mathrm{kj}}(\mathrm{t})+\mu \mathrm{W}^{\mathrm{o}}{ }_{\mathrm{kj}}(\mathrm{t})+\left(\eta \text {. Modified } \delta^{\mathrm{o}}{ }_{\mathrm{pk}} \cdot i_{p j}\right)
$$

9. Update weights on the hidden layer.

$\mathrm{W}^{\mathrm{o}}{ }_{\mathrm{ji}}(t+1)=\mathrm{W}_{\mathrm{ji}}^{\mathrm{h}}(\mathrm{t})+\left(\eta \cdot\right.$ Modified $\left.\delta^{\mathrm{h}}{ }_{\mathrm{pj}} \cdot X_{\mathrm{i}}\right) 3.5$

where $\eta$ is the learning rate. Repeat steps from step 1 tostep 9 until the error $\left(Y_{p k}-O_{p k}\right)$ was acceptably small foreach of the training vector pair. The developed algorithm isstopped when the cubes of the differences between theactual and target values summed over units and all patterns are acceptably small.where net ${ }_{\mathrm{pj}}^{\mathrm{h}}$ is net input to the $j$ th hidden unit, $\mathrm{i}_{\mathrm{pj}}$ is net input to the $j$ th hidden unit, net ${ }^{0}{ }_{\mathrm{pk}}$ is net input to the $k$ th output unit, $\mathrm{W}^{\mathrm{o}}{ }_{\mathrm{kj}}$ weight on the connection from the $j$ th hidden unit to $k$ th output unit, $\mathrm{f}^{\prime}$ is derivative of activation function, $\delta_{\mathrm{pk}}^{\mathrm{o}}$ is signal error term for the $k$ th output unit and $\mathrm{t}_{1}$ is desired output

\subsection{Classification and Testing Stage}

The classification phase is the last stage of the developed character recognition system. This phase determined the overall performance of the developed algorithm. Three types of classifier were implemented for online character recognition.
(a) $\mathrm{C} 1$ (MGA and MOBP)
(b) $\mathrm{C} 2$ (MGABased MOBP)
(c) C3 (MGA and MGABased MOBP).

Three classifiers ( $\mathrm{C} 1, \mathrm{C} 2$ and $\mathrm{C} 3$ ) were formulated from MGA-MOBP such that $\mathrm{C} 1$ classified without using MGA at classification level, $\mathrm{C} 2$ classified using MGA at classification level while C3 employed MGA at feature selection level and classified at classification level. The performance of each of $\mathrm{C} 1, \mathrm{C} 2$ and $\mathrm{C} 3$ was evaluated using recognition failure, training time and correct recognition accuracy. The best of the three classifiers was determined on the basis of correct training time and recognition accuracy. The objective for using the optimization algorithm at feature selection level is in twofold: (i) feature reduction which improves the convergence time and (ii) elimination of insignificant features which improves the classification accuracy and reduce computation time imposed of whole features. In this research work modified genetic algorithm was used for optimal feature selection. MGA was used during the training to solve the problem of local minima in backpropagation and finds a nearly globally optimal set weights on a relatively short time.

\section{CONCLUSION AND FUTURE WORK}

This research developed a hybrid of structural and statistical features used for extracting features from character images. This was developed to solve the problem of poor feature extraction and selection of online character recognition system. A model for online character recognition was proposed using MGA and MOPB algorithm for three classifiers: $\mathrm{C} 1, \mathrm{C} 2$ and $\mathrm{C} 3$ for overall recognition accuracy. MGA was also used for feature selection to reduce the feature space which enhanced recognition accuracy and reduced training time. Future and further research work may be geared towards investigating the performance of the model based on training time, recognition time and recognition accuracy.

\section{REFERENCES}

[1] Agnihotri, V. P. (2012): "Offline Handwritten Devanagari Script Recognition”, Information Technology and Computer Science, (8): 37-42.

[2] Ashutosh, A, R. Rajneesh and RenuDhir (2012): "Handwritten Devanagari Character Recognition Using Gradient Features,'International Journal of Advanced Research in Computer Science and Software Engineering, (2)5.

[3] Ayyaz, M. N., Javed, I. and Mahmood, W. (2012): "Handwritten Character Recognition Using Multiclass SVM Classification with Hybrid Feature Extraction", Pak. J. Engg. \& Appl. Sci. 10: 57-67.

[4] Fenwa, O D, Omidiora, E, O and Fakolujo, O. A. (2012): "Development of a Feature Extraction Technique for Online Character Recognition System", Innovative System Design and Engineering ISSN 22222871(Online) 3(3): 10-23

[5] Fenwa, O. D., Omidiora, E, O., Fakolujo, O. A., Ajala, F. A., Oke, A. O. (2012): “A Modified Optical Backpropagation Neural Network Model in Online Handwritten Character Recognition System", International Journal of Computer Application 2 4(2): 190-201.

[6] Haykin, S. (2003): "Neural Networks: A comprehensive Foundation", PHI New-Delhi, India

[7] Ibrahim A. Adeyanju, Olusayo D. Fenwa, Elijah O. Omidiora(2014): "Effect of Non-image Features on recognition of handwritten alpha-numeric characters" International Journal of Computers and Technology(IJCT).13(11):5155-5161.

[8] Jumanal Shilpa and Holi, Ganga (2013): "On-line Handwritten English Character Recognition Using Genetic Algorithm" International Journal of Computer Trends and Technology (IJCTT). 4(6): 1885-1890.

[9] Muhammad, F. Z., Dzulkifli, M. and Razib, M. O. (2006): "Writer Independent Online Handwritten Character Recognition Using a simple Approach", Information Technology Journal 5(3): 476-484.

[10] Omidiora E. O., Oyediran, G. O., Olabiyisi, S. O. and Arulogun, O. T. (2008): "Classification of Soils of Central Western Nigeria using Neural Network Rule 
Extraction and Decision Table, Agricultural Journal 3(4):305-312

[11] Omidiora, E. O., Oladipo, O., Oyeleye ,C. A and Ismaila W. O. (2013) A Study of Genetic Principal Component Analysis (GPCA)in feature extraction and recognition of face images, Journal of Computer Science And Engineering Vol. 19, issue 1 ...

[12] Omidiora, E. O., Adeyanju, I. A. Oladipo., Fenwa, O. D. (2014) "Comparison of Machine Learning Classifiers For Recognition of Face Images", Journal Of Computer Science And Engineering 19(1):1-5

[13] Padhi, D (2012):"Novel Hybrid Approach for Odia handwritten Character Recognition System", International Jornal of Advanced Research in Computer Science and Software Engineering, 2(5):150-157.

[14] Pradeep, J., Srinivasan, E. and Himavathi, S. (2011): "Diagonal Based Feature Extraction for handwritten
Alphabets Recognition system using Neural Network", International Journal of Computer Science \& Information Technology (IJCSIT), 3(1): 27-38.

[15] Ranpreet, K. and Singh, B. (2011): "A Hybrid Neural Approach for Character Recognition System”, (IJCSIT) International Journal of Computer Science and Information Technologies, 2 (2):721-726.

[16] Razzak, M. I., Hussain, S.A. and Mirza,A.A(2012):"BioInspired Multilayered and Multilanaguage Arabic Script Character Recognition System", International Journal of Innovative Computing, Information and Control . 6(4):2681-2691.

[17] Yeremia Hendy, Niko Adrianus Yuwono, Pius Raymond and Widodo Budiharto (2013): "Genetic Algorithm and Neural Network for Optical character recogn ition" Journal of computer science 9 (11): 1435-1442. 